\title{
Detection of small-size planetary candidates with CoRoT data
}

\author{
Aldo S. Bonomo ${ }^{1}$, Pierre-Yves Chabaud ${ }^{1}$, Magali Deleuil ${ }^{1}$, \\ Claire Moutou ${ }^{1}$ and Pascal Bordé ${ }^{2}$ \\ ${ }^{1}$ Laboratoire d'Astrophysique de Marseille, 38 rue Frédéric Joliot-Curie, 13388 Marseille Cedex \\ 13, France \\ email: aldo.bonomo@oamp.fr \\ ${ }^{2}$ Institut d'Astrophysique Spatiale, centre universitaire d'Orsay Bât 120-121, 91405 ORSAY \\ CEDEX
}

\begin{abstract}
With the discovery of CoRoT-7b, the first transiting super-Earth, the CoRoT space mission has shown the capability to detect short-period rocky planets around solar-like stars. By performing a blind test with real CoRoT light curves, we want to establish the detection threshold of small-size planets in CoRoT data. We investigate the main obstacles to the detection of transiting super-Earths in CoRoT data, notably the presence of short-time scale variability and hot pixels.
\end{abstract}

Keywords. methods: data analysis, techniques: photometric, stars: late-type

\section{Introduction}

The CoRoT space mission searches for planetary transits by monitoring the optical flux of thousands of stars in several fields of view. It has recently led to the discovery of CoRoT-7b, the first transiting super-Earth (Léger et al. 2009). By simulating transits of super-Earths and Neptunes in real CoRoT light curves and searching for them blindly, we want to investigate the capability of CoRoT to detect small-size planets, super-Earths and Neptunes, in short-period orbits $(P<10$ days $)$.

\section{Simulations and data analysis}

We chose 500 real light curves of the CoRoT long run LRa01 which lasted 131.5 days. Central transits by super-Earths with orbital period $P$ between 1 and 15 days were simulated in 100 of the aforementioned light curves with visual magnitude $11<V<14.5$. In as many light curves with $12<V<16$, central transits by Neptune-like planets were inserted. For simplicity, the parent star was assumed in all cases to be a Sun-like star $\left(R_{*}=R_{\odot}\right.$ and $\left.M_{*}=M_{\odot}\right)$.

The simulated transits by super-Earths and Neptunes were searched for blindly, i.e. without knowing in advance in which light curves they had been inserted, by means of the LAM transit detection pipeline (Bonomo et al. , in preparation). It foresees the following steps: a) a 5-sigma clipping to filter out outliers due to proton impacts during the passage of the satellite at the South Atlantic Anomaly; b) a high-pass sliding median filter to remove stellar variability; c) a Savitzky-Golay low-pass filter to remove high-frequency variations of instrumental origin; d) an automatic detection and correction of the most evident hot pixels; e) the search for transits by means of the BLS algorithm (Kovács et al. 2002) with the directional correction. 


\section{Results and discussion}

The percentage of detected super-Earths with $P<10$ days around stars with $11<$ $V<13.5$ is $35 \% .78 \%$ of Neptunes with $P<10$ days and $V<15$ were correctly found. Figure 1 shows the depth of the simulated transits as a function of their Signal-to-Noise Ratio (SNR). Most of the discovered transits have $S N R \geqslant 11$. This shows that CoRoT is able to detect super-Earths with a SNR much lower than CoRoT-7b (Fig. 2). We had no false positives.

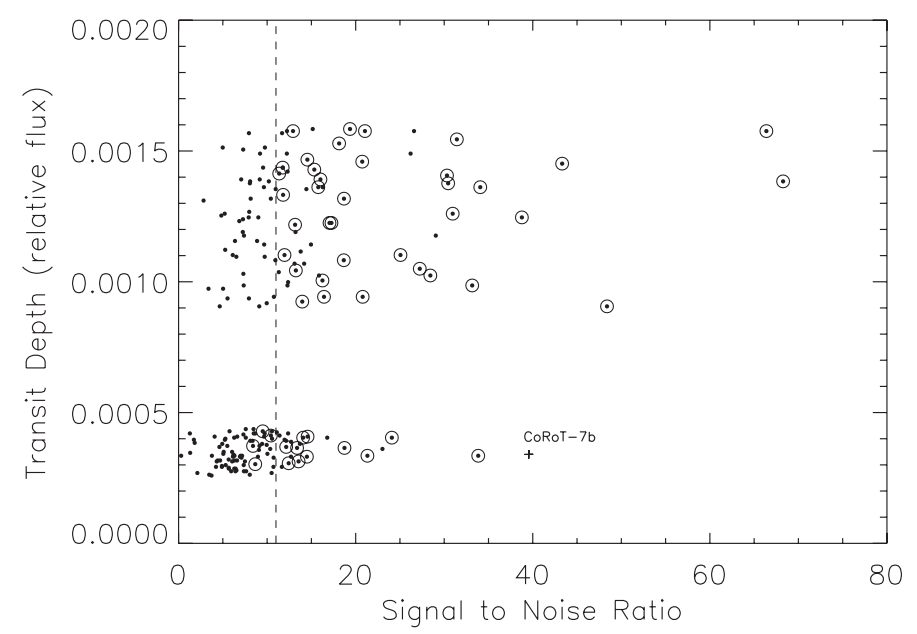

Figure 1. Depth of the simulated transits vs their Signal-to-Noise Ratio. Vertical dashed line: empirical detection limit at $S N R \sim 11$. The cross indicates the position of CoRoT-7b.

All the undiscovered transits with $S N R>15$ were not detected because of short timescale $(<1.5$ days) variability affecting the light curves in which they had been simulated. The undiscovered transits with $11<S N R<15$ went undetected because of short timescale variability or, more often, the presence of hot pixels. Indeed, the latter hinder the search for shallow planetary transits since, after the filtering of stellar variability, they give rise to artificial dips in the residuals that can be erroneously identified as transits by the detection algorithms.

Starting from the aforementioned results, work is in progress to infer some statistics on the presence of low-mass planets in the CoRoT fields of view. Indeed, it is an intriguing issue to understand if the detection of only one low-mass planet by the CoRoT team (e.g., CoRoT-7b) is compatible with the occurrence rate given by radial velocity surveys (Howard et al. 2010; Mayor et al. 2009), after taking both the transit probability and the detection rate derived from our blind test into account. Both radial velocity survey (Howard et al. 2010; Mayor et al. 2009) and theoretical core accretion models (e.g., Mordasini et al. 2009) indicate a a pile up of planets in the Neptunian and, even more, super-Earth mass domain.

\section{References}

Howard, A. W., Marcy, G. W., Johnson, J. A., et al. 2010, Science, 330, 653

Kovács, G., Zucker, S., \& Mazeh, T. 2002, A\& $A$, 391, 369

Léger, A., Rouan, D., Schneider, J., et al. 2009, A\&A, 506, 287

Mayor, M., Udry, S., Lovis, C., et al. 2009, A\& $A$, 493, 639

Mordasini, C., Alibert, Y., Benz, W., \& Naef, D. 2009, A\& $A, 501,1161$ 